PROCEEDINGS OF THE

AMERICAN MATHEMATICAL SOCIETY

Volume 125, Number 6, June 1997, Pages 1785-1792

S $0002-9939(97) 03759-3$

\title{
WEAK SOLUTIONS OF PARABOLIC EQUATIONS IN NON-CYLINDRICAL DOMAINS
}

\author{
RUSSELL M. BROWN, WEI HU, AND GARY M. LIEBERMAN \\ (Communicated by Jeffrey B. Rauch)
}

\begin{abstract}
In their classical work, Ladyzhenskaya and Ural'tseva gave a definition of weak solution for parabolic equations in cylindrical domains. Their definition was broad enough to guarantee the solvability of all such problems but narrow enough to guarantee the uniqueness of these solutions. We give here some alternative definitions which are appropriate to non-cylindrical domains, and we prove the unique solvability of such problems.
\end{abstract}

\section{INTRODUCTION}

In [1], Ladyzhenskaya and Ural'tseva defined a weak solution for parabolic equations in divergence form with bounded measurable coefficients:

$$
-u_{t}+D_{i}\left(a^{i j} D_{j} u+b^{i} u\right)+c^{i} D_{i} u+c^{0} u=D_{i} f^{i}+g \text { in } \Omega
$$

where $\Omega=\omega \times(0, T)$ for some domain $\omega \subset \mathbb{R}^{n}$. It is also possible to adjoin initial and (zero) boundary values to this equation. To do so, we set $B \Omega=\omega \times\{0\}$, $S \Omega=\partial \Omega \times(0, T), \omega(\tau)=\omega \times\{\tau\}$, and $\Omega(\tau)=\omega \times(0, \tau)$. We then say that $u$ is a weak solution of (1.1a) and

$$
u=0 \text { on } S \Omega, u=\varphi \text { on } B \Omega
$$

for square integrable $f, g$, and $\varphi$ if $u$ is a function in

$$
V_{0}=C^{0}\left([0, T] ; L^{2}(\omega)\right) \cap L^{2}\left([0, T] ; H_{0}^{1}(\omega)\right)
$$

satisfying the integral identity

$$
\begin{aligned}
0=\int_{\Omega(\tau)} u \psi_{t} d X & -\int_{\Omega(\tau)}\left(a^{i j} D_{j} u+b^{i} u-f^{i}\right) D_{i} \psi d X \\
& +\int_{\Omega(\tau)}\left(c^{i} D_{i} u+c^{0} u-g\right) \psi d X+\int_{B \Omega} \varphi \psi d x-\int_{\omega(\tau)} u \psi d x
\end{aligned}
$$

for all $\tau \in(0, T)$ and all $\psi \in C^{1}(\bar{\Omega})$ which vanish on $S \Omega$. (Here and below $X=$ $(x, t)$ denotes a point in $\mathbb{R}^{n+1}$.) As long as the coefficients $a^{i j}, b^{i}, c^{i}$, and $c^{0}$ are bounded measurable functions and the matrix $\left(a^{i j}\right)$ is uniformly positive definite in $\Omega$, Ladyzhenskaya and Ural'tseva show that (1.1) has a unique solution.

Received by the editors August 4, 1995 and, in revised form, January 2, 1996.

1991 Mathematics Subject Classification. Primary 35K15; Secondary 35D05.

The first author was supported in part by the NSF and the Commonwealth of Kentucky through the NSF-EPSCoR program. 
A key element in their proof is the energy inequality

$$
\sup _{\tau} \int_{\omega(\tau)} u^{2} d x+\int_{\Omega}|D u|^{2} d X \leq C\left(\int_{\Omega}|f|^{2}+g^{2} d X+\int_{B \Omega} \varphi^{2} d x\right),
$$

where the constant $C$ is determined only by the above indicated $L^{\infty}$ norms, the dimension $n$ and an upper bound for $T$. (In fact, the conditions on the coefficients $b^{i}, c^{i}$, and $c^{0}$ can be relaxed to their membership in suitable $L^{p, q}$ spaces, and the function $g$ need only lie in some other $L^{p, q}$ space. We shall return to this point in detail in Section 5.)

The techniques involved in proving these results are very closely connected to the special form of the domain $\Omega$ as a cylinder. If $u$ is smooth enough, then (1.3) is a simple consequence of (1.2) since one can replace the test function $\psi$ by $u$ and then integrate the quantity $u u_{t}$ explicitly. For $u \in V_{0}$, the test function involves the Steklov average of $u$ :

$$
u_{h}(x, t)=\frac{1}{h} \int_{0}^{h} u(x, t+\tau) d \tau
$$

and this expression leads to a suitable test function only if $\Omega$ is cylindrical. In addition, the form of the equation (1.1a) precludes any simple functional-analytical approach to existence and uniqueness since not every element of $V_{0}$ is a solution of an equation of the form (1.1a). Generally, functions in $V_{0}$ have time derivatives which cannot be written as the sum of an $L^{2}$ function and the spatial derivative of an $L^{2}$ function, and the set of $V_{0}$ functions which have time derivatives of this special form is dense, and hence not closed, in $V_{0}$. Hence, the extension of Ladyzhenskaya and Ural'tseva's results is more subtle than one might expect.

Although there is a large literature on classical solutions of parabolic equations in non-cylindrical domains (see the references in [4], for example), we know of only a few other papers dealing with weak solutions of parabolic equations in noncylindrical domains. Lions [5] proved existence and uniqueness of weak solutions for a large class of higher order equations and systems, but his class of domains is much smaller than ours, as he only considers domains which can be written locally as the graph of a $C^{\infty}$ function. Yong [6] considers a broader class of domains than we do, but he assumes that the functions $f^{i}$ and $g$ are in a smaller space so that the weak solutions are known to be continuous. This additional regularity is not needed for our proof. Finally, Cannarsa, Da Prato, and Zolèsio [2] recast the problem in a semigroup setting. Their hypotheses on $\Omega$ are more restrictive than ours and an energy inequality is not proved because the authors look in a larger class of functions for a weak solution. (On the other hand, their method also applies to hyperbolic equations.)

In this paper we show that problem (1.1) has a unique weak solution in the space $V_{0}$ (defined below and which is the same as $V_{0}$ defined above when $\Omega$ is cylindrical) when $\mathcal{P} \Omega \in H_{1}$; this condition is the parabolic analog of $\Omega$ being a bounded Lipschitz domain for elliptic equations. Section 2 presents definitions and gives a precise statement of our result. The energy inequality is proved in Section 3 , and Section 4 uses this energy inequality to show that (1.1) has a unique weak solution. Finally Section 5 contains a discussion of some extensions of our main result. 


\section{Statement of Results}

As previously mentioned, we write $X=(x, t)=\left(x^{1}, \ldots, x^{n}, t\right)$ for a point in $R^{n+1}$ with $n$ a positive integer. We also write $X_{0}=\left(x_{0}, t_{0}\right)$ and $Y=(y, s)$ for other points in $\mathbb{R}^{n+1}$, and we define a metric on $\mathbb{R}^{n+1}$ by

$$
|X-Y|=\left(|x-y|^{2}+|t-s|\right)^{1 / 2}
$$

(Although we use the same symbol to denote the absolute value of an element in $\mathbb{R}$, the Euclidean length of a vector in $\mathbb{R}^{n}$ and this metric in $\mathbb{R}^{n+1}$, it will be clear from the context which of these meanings is relevant.) It will also be useful to write $X^{\prime}$ for the point $\left(x^{1}, \ldots, x^{n-1}, t\right)$ in $\mathbb{R}^{n}$. We use $Q\left(X_{0}, r\right)$ to denote the cylinder $\left\{\left|x-x_{0}\right|<r, t_{0}-r^{2}<t<t_{0}\right\}$.

For $\Omega$, a connected open subset of $\mathbb{R}^{n+1}$, we define $\omega(\tau)=\{(x, \tau) \in \Omega\}, \Omega(\tau)=$ $\{X \in \Omega: t<\tau\}$, and $I(\Omega)=\{\tau \in \mathbb{R}: \Omega(\tau) \neq \emptyset\}$. We also define the parabolic boundary of $\Omega, \mathcal{P} \Omega$, to be the set of all points $X_{0} \in \partial \Omega$ such that the cylinder $Q\left(X_{0}, r\right)$ contains points not in $\Omega$ for any $r>0$. We write $B \Omega$ for the set of all points $X_{0} \in \mathcal{P} \Omega$ such that $Q\left(\left(x_{0}, t+r^{2}\right), r\right) \subset \Omega$ for some $r>0$, and $S \Omega$ for $\mathcal{P} \Omega \backslash B \Omega$. The function $d$ is defined in $\Omega$ by

$$
d(X)=\min _{\substack{Y \in \mathcal{S} \Omega \\ s \leq t}}|X-Y|
$$

For any open subset $\Sigma$ of $\mathbb{R}^{n+1}$, we write $H_{1}(\Sigma)$ for the set of all functions $f$ defined on $\Sigma$ such that there is a nonnegative constant $F$ for which $|f(X)-f(Y)| \leq$ $F$ for all $X$ and $Y$ in $\Sigma$. The infimum of all such constants is the $H_{1}$ norm of $f$. (In particular, $f$ will be uniformly continuous on $\Sigma$ and uniformly Lipschitz with respect to $x$.)

We say that $\mathcal{P} \Omega \in H_{1}$ if there is a positive constant $\delta$ such that for any point $X_{0} \in S \Omega$, there is a function $f \in H_{1}\left(\left\{\left|X^{\prime}-X_{0}^{\prime}\right|<\delta\right\}\right.$ ) for which (after a suitable rotation of $x$-axes)

$$
\Omega \cap\left\{\left|X-X_{0}\right|<\delta\right\}=\left\{\left|X-X_{0}\right|<\delta, x^{n}>f\left(x^{\prime}, t\right), t \in I(\Omega)\right\}
$$

and if $B \Omega$ lies in a single hyperplane $\left\{t=t_{0}\right\}$.

When $\mathcal{P} \Omega \in H_{1}$, we define $C_{S}^{1}(\Omega)$ to be the set of all functions $u$ defined on $\bar{\Omega}$ such that $u, D u$, and $u_{t}$ are uniformly continuous on $\Omega$ with $u=0$ on $S \Omega$. We then define the norm

$$
\|u\|_{V}=\sup _{\tau \in I(\Omega)} \int_{\omega(\tau)} u^{2} d x+\int_{\Omega(\tau)}|D u|^{2} d X
$$

and use $V_{0}$ to denote the closure of $C_{S}^{1}(\Omega)$ with respect to this norm. It is simple to verify that this definition leads to the space denoted by $V_{0}$ in the introduction when $\Omega$ is a cylinder.

Finally, we say that $u$ is a weak solution of (1.1) if $u \in V_{0}$ and if the integral identity (1.2) holds for all $\psi \in C_{S}^{1}(\Omega)$. With these definitions, our result can be written in the following form: 
Theorem 1. Let $\mathcal{P} \Omega \in H_{1}$ and suppose that $a^{i j}, b^{i}, c^{i}$, and $c^{0}$ are $L^{\infty}(\Omega)$ functions such that

$$
\begin{gathered}
\left\|a^{i j}\right\|_{\infty} \leq \Lambda, \\
\left\|b^{i}-c^{i}\right\|_{\infty} \leq \Lambda_{1}, \\
c^{0} \leq \Lambda_{1} \text { a.e. in } \Omega,
\end{gathered}
$$

for some nonnegative constants $\Lambda$ and $\Lambda_{1}$. Suppose also that there is a positive constant $\lambda$ such that

$$
a^{i j} \xi_{i} \xi_{j} \geq \lambda|\xi|^{2}
$$

for all $\xi \in \mathbb{R}^{n}$ and almost all $x \in \Omega$. Then for any $f$ and $g$ in $L^{2}(\Omega)$ and any $\varphi \in L^{2}(B \Omega)$, there is a unique weak solution $u$ of (1.1). Moreover, there is a constant $C$ determined only by $n, \lambda, \Lambda, \Lambda_{1}$, and the length of $I(\Omega)$ such that (1.3) holds.

\section{The ENERGy INEQUALITY}

To prove our energy inequality, we introduce a regularized distance. According to [3, Section 3], if $\mathcal{P} \Omega \in H_{1}$, then there is a function $\rho \in H_{1}(\Omega) \cap C^{\infty}(\Omega)$ with $d / 2 \leq \rho \leq 2 d,\left|D^{2} \rho\right|+\left|\rho_{t}\right| \leq C / d,\left|D \rho_{t}\right| \leq C d^{-2}$. Moreover, in any set $\Omega_{\delta}=$ $\Omega \cap\left\{\left|X-X_{0}\right|<\delta\right\}$ from the definition of $\mathcal{P} \Omega \in H_{1}$, we have that $1 / 2 \leq D_{n} \rho \leq 2$. Hence we can introduce new coordinates $Y$ in $\Omega_{\delta}$ by

$$
y^{\prime}=x^{\prime}, y^{n}=\rho(X), s=t,
$$

and we note that $\operatorname{det}(\partial X / \partial Y)=1 / D_{n} \rho$. In the rest of this section, we use $D_{i}$ for derivatives in the original coordinates, $x, t$, and $\partial_{i}$ for derivatives in the new coordinates, $y, s$. Also, we infer from direct calculation that $\psi_{t}=\psi_{s}+\frac{\partial \psi}{\partial y^{n}} \rho_{t}$ for any sufficiently smooth function $\psi$.

We write $Q^{+}$for the image of $\Omega_{\delta}$ in $Y$-coordinates, so that Hardy's inequality implies that

$$
\int_{Q^{+}}\left(\frac{u}{y^{n}}\right)^{2} d Y \leq C \int_{Q^{+}}|\partial u|^{2} d Y \leq C\|u\|_{V}^{2} .
$$

If $\psi \in C_{S}^{1}$ is zero outside $\Omega_{\delta}$ and if $I\left(\Omega_{\delta}\right)=\left(\tau_{1}, \tau_{2}\right)$, it follows that (1.2) can be written in $Y$-coordinates as

$$
\begin{aligned}
\int_{Q^{+}} u \psi_{s} \frac{1}{D_{n} \rho} d Y & +\int_{Q^{+}} u \partial_{n} \psi \frac{\rho_{t}}{D_{n} \rho} d Y-\int_{Q^{+}}\left(A^{i j} \partial_{j} u+B^{i} u-F^{i}\right) \partial_{i} \psi \frac{1}{D_{n} \rho} d Y \\
& +\int_{Q^{+}}\left(C^{i} \partial_{i} u+c^{0} u-g\right) \psi \frac{1}{D_{n} \rho} d Y \\
& =\int_{B^{+}\left(\tau_{1}\right)} u \psi \frac{1}{D_{n} \rho} d y-\int_{B^{+}\left(\tau_{2}\right)} u \psi \frac{1}{D_{n} \rho} d y
\end{aligned}
$$

where $\partial_{i}=\partial / \partial y^{i}$,

$$
A^{i j}=a^{k m} D_{k} y^{i} D_{m} y^{j}, B^{i}=b^{k} D_{k} y^{i}, C^{i}=c^{k} D_{k} y^{i},
$$

and $B^{+}(\tau)=\left\{(y, \tau) \in \overline{Q^{+}}\right\}$. 
Next, we define

$$
w_{h}(Y)=\frac{1}{h} \int_{s}^{s+h} w(y, \tau) d \tau
$$

for $h \in \mathbb{R}$ and any function $w$. As in [1, Section III.1] but also taking Hardy's inequality into account, we may use the test function $\psi=\zeta_{-h}$ for $h$ sufficiently small, and $\zeta$ a $C_{S}^{1}\left(\overline{\Omega_{\delta}}\right)$ function which vanishes on $\partial \Omega_{\delta} \cap \Omega$ and for $t=\tau_{1}$. It then follows that

$$
\begin{aligned}
-\int_{Q^{+}} \zeta\left(\frac{u}{D_{n} \rho}\right)_{h, s} d Y & +\int_{Q^{+}} \partial_{n} \zeta\left(\frac{u \rho_{t}}{D_{n} \rho}\right)_{h} d Y \\
& -\int_{Q^{+}}\left(\left(A^{i j} \partial_{j} u+B^{i} u-F^{i}\right) / D_{n} \rho\right)_{h} \partial_{i} \zeta d Y \\
& +\int_{Q^{+}}\left(\left(C^{i} \partial_{i} u+c^{0} u-g\right) / D_{n} \rho\right)_{h} \zeta d Y=0 .
\end{aligned}
$$

The approximation argument on [1, p. 142] (and Hardy's inequality) then shows that this identity holds for any $\zeta \in V_{0}\left(Q^{+} \cap\left\{t<\tau_{2}-h\right\}\right)$. In particular, we can choose $\zeta=u_{h} \eta$ for $\eta \in C^{1}\left(\overline{\Omega_{\delta}}\right)$ a nonnegative function which vanishes on $\partial \Omega_{\delta} \cap \Omega$. Sending $h \rightarrow 0$, we find that

$$
\begin{aligned}
\frac{1}{2} \int_{B^{+}\left(\tau_{2}\right)} u^{2} \eta \frac{1}{D_{n} \rho} d y & -\frac{1}{2} \int_{B^{+}\left(\tau_{1}\right)} u^{2} \eta \frac{1}{D_{n} \rho} d y \\
& -\int_{Q^{+}}\left(A^{i j} \partial_{i} u+B^{i} u-F^{i}\right) \partial_{i}(u \eta) \frac{1}{D_{n} \rho} d Y \\
& +\int_{Q^{+}}\left[\left(C^{i} \partial_{i} u+c^{0} u-g\right) u \eta\right] \frac{1}{D_{n} \rho} d Y=I,
\end{aligned}
$$

where

$$
\begin{array}{r}
I=-\frac{1}{2} \int_{Q^{+}} \eta \partial_{n}\left(u^{2}\right) \frac{\rho_{t}}{D_{n} \rho} d Y-\int_{Q^{+}} u^{2} \partial_{n} \eta \frac{\rho_{t}}{D_{n} \rho} d Y \\
-\frac{1}{2} \int_{Q^{+}} u^{2} \eta_{s} \frac{1}{D_{n} \rho} d Y+\frac{1}{2} \int_{Q^{+}} u^{2} \eta\left(\frac{1}{D_{n} \rho}\right)_{s} d Y .
\end{array}
$$

We now rewrite $I$. First we integrate by parts to see that

$$
\int_{Q^{+}} \eta \partial_{n}\left(u^{2}\right) \frac{\rho_{t}}{D_{n} \rho} d Y=-\int_{Q^{+}} u^{2}\left[\partial_{n} \eta \frac{\rho_{t}}{D_{n} \rho}+\eta \partial_{n}\left(\frac{\rho_{t}}{D_{n} \rho}\right)\right] d Y
$$

and hence

$$
\begin{aligned}
I & =\frac{1}{2} \int_{Q^{+}} u^{2}\left[-\partial_{n} \eta \rho_{t}-\eta_{s}\right] \frac{1}{D_{n} \rho} d Y \\
& +\frac{1}{2} \int_{Q^{+}} u^{2} \eta\left[\partial_{n}\left(\frac{\rho_{t}}{D_{n} \rho}\right)+\left(\frac{1}{D_{n} \rho}\right)_{s}\right] d Y .
\end{aligned}
$$

We denote these two integrals by $I_{1}$ and $I_{2}$. In $I_{1}$, we note that $\eta_{t}=\partial_{n} \eta \rho_{t}+\eta_{s}$, so

$$
I_{1}=-\int_{Q^{+}} u^{2} \eta_{t} \frac{1}{D_{n} \rho} d Y \text {. }
$$

For $I_{2}$, we note that

$$
\partial_{n}\left(\frac{\rho_{t}}{D_{n} \rho}\right)=\frac{1}{D_{n} \rho} \partial_{n}\left(\rho_{t}\right)+\rho_{t} \partial_{n}\left(\frac{1}{D_{n} \rho}\right)=\frac{1}{\left(D_{n} \rho\right)^{2}} D_{n t} \rho+\rho_{t} \partial_{n}\left(\frac{1}{D_{n} \rho}\right),
$$


and that

$$
\left(\frac{1}{D_{n} \rho}\right)_{s}=\left(\frac{1}{D_{n} \rho}\right)_{t}-\rho_{t} \partial_{n}\left(\frac{1}{D_{n} \rho}\right)
$$

so $I_{2}=0$. Therefore

$$
I=-\frac{1}{2} \int_{Q^{+}} u^{2}\left(\eta^{2}\right)_{t} \frac{1}{D_{n} \rho} d Y
$$

from which it follows that

$$
\begin{aligned}
\frac{1}{2} \int_{\omega\left(\tau_{2}\right)} u^{2} \eta d x & -\frac{1}{2} \int_{\omega\left(\tau_{1}\right)} u^{2} \eta d x-\int_{\Omega(\tau)}\left(a^{i j} D_{j} u+b^{i} u-f^{i}\right) D_{i}(u \eta) d X \\
& +\int_{\Omega(\tau)}\left[\left(c^{i} D_{i} u+c^{0} u-g\right) u \eta\right] d X=-\frac{1}{2} \int_{\Omega(\tau)} u^{2} \eta_{t} d X .
\end{aligned}
$$

Now we cover $S \Omega$ by a finite number of cylinders $Q(1), \ldots, Q(N)$ of the form $\Omega_{\delta}$. Then there is a constant $\varepsilon>0$ such that $d>\varepsilon$ in $\Omega \backslash \cup Q(k)$, so if we write $Q(0)$ for $\{d>\varepsilon / 2\}$, then $Q(0), \ldots, Q(N)$ is a finite open cover of $\Omega \cup \mathcal{P} \Omega$. Using a subordinate partition of unity $\left(\eta_{k}\right)_{0 \leq k \leq N}$, we infer this inequality for each $\eta_{k}$. (Of course, the proof for $k=0$ is simpler because we do not need to change variables.) Summing on $k$, we find that

$$
\begin{aligned}
\frac{1}{2} \int_{\omega\left(t_{1}\right)} u^{2} d x & -\frac{1}{2} \int_{B \Omega} \varphi^{2} d x-\int_{\Omega}\left(a^{i j} D_{j} u+b^{i} u-f^{i}\right) D_{i} u d X \\
& +\int_{\Omega}\left[\left(c^{i} D_{i} u+c^{0} u-g\right) u\right] d X=0
\end{aligned}
$$

where $I(\Omega)=\left(t_{0}, t_{1}\right)$, and repeating the argument with $\Omega(\tau)$ in place of $\Omega$ shows that

$$
\begin{aligned}
\frac{1}{2} \int_{\omega(\tau)} u^{2} d x & -\frac{1}{2} \int_{B \Omega} \varphi^{2} d x-\int_{\Omega(\tau)}\left(a^{i j} D_{j} u+b^{i} u-f^{i}\right) D_{i} u d X \\
& +\int_{\Omega(\tau)}\left[\left(c^{i} D_{i} u+c^{0} u-g\right) u\right] d X=0
\end{aligned}
$$

for any $\tau \in I(\Omega)$. It now follows from hypotheses $(2.1 \mathrm{a}-\mathrm{c})$ that

$$
\int_{\omega(\tau)} u^{2} d x+\int_{\Omega(\tau)}|D u|^{2} \leq C\left(\int_{\Omega(\tau)} u^{2} d X+\|f\|^{2}+\|g\|^{2}+\|\varphi\|^{2}\right),
$$

and hence

$$
\int_{\omega(\tau)} u^{2} d x \leq C \int_{\Omega(\tau)} u^{2} d X+C K
$$

where

$$
K=\|f\|^{2}+\|g\|^{2}+\|\varphi\|^{2} .
$$

It then follows from Gronwall's inequality that

$$
\int_{\Omega(\tau)} u^{2} d X \leq C K
$$

and then (3.1) gives (1.3). 


\section{Proof of Theorem 1}

It follows from the energy inequality in Section 3 that any weak solution of (1.1) must be unique, so we only have to show that a weak solution exists. To this end, we recall that, for $\alpha \in(0,1)$, we say that $f \in H_{1+\alpha}(\Sigma)$ if $f \in H_{1}(\Sigma)$ with the additional assumptions that

$$
|D f(X)-D f(Y)| \leq C|X-Y|^{\alpha}
$$

for all $X$ and $Y$ in $\Sigma$ and

$$
|f(X)-f(Y)| \leq C|X-Y|^{1+\alpha}
$$

for $X$ and $Y$ in $\Sigma$ with $x=y$. With the obvious definition of $\mathcal{P} \Omega \in H_{1+\alpha}$, we know from $[4$, Theorem 11.3] that there is a unique (classical) solution of the equation

$$
-u_{t}+a^{i j} D_{i j} u+B^{i} D_{i} u+c u=F \text { in } \Omega,
$$

with boundary condition

$$
u=\Phi \text { on } \mathcal{P} \Omega
$$

if the coefficients $a^{i j}, B^{i}, c$, and $F$ are sufficiently smooth (for example, $H_{1}$ ) and if $\Phi \in H_{1+\alpha}$ and $\mathcal{P} \Omega \in H_{1+\alpha}$. In addition, $u \in H_{1+\alpha}(\Omega)$, and hence $u$ is also a weak solution of (1.1) with suitable $B^{i}, c$ and $F$ provided the coefficient functions in (1.1) and $\mathcal{P} \Omega$ are sufficiently smooth.

Now we take sequences of smooth functions $\left(a_{m}^{i j}\right),\left(b_{m}^{i}\right),\left(c_{m}^{i}\right)$, and $\left(c_{m}^{0}\right)$ converging a.e. to $a^{i j}, b^{i}, c^{i}$, and $c^{0}$ respectively and satisfying $(2.1 \mathrm{a}-\mathrm{c})$ uniformly. We also take sequences of smooth functions $\left(f_{m}^{i}\right),\left(g_{m}\right)$, and $\left(\varphi_{m}\right)$ converging in $L^{2}$ to $f^{i}$, $g$, and $\varphi$, respectively so that $\varphi_{m}$ is compactly supported in $B \Omega$. We then choose $\varepsilon(m)>0$ so that $\varphi(X)=0$ if $\rho(X)<\varepsilon(m)$ and set $\Omega_{m}=\{X \in \Omega: \rho(X)>\varepsilon(m)\}$. It follows from the remarks in the preceding paragraph that, for each $m$, there is a classical solution $u_{m}$ of

$$
\begin{gathered}
-u_{m, t}+D_{i}\left(a_{m}^{i j} D_{j} u_{m}+b_{m}^{i} u_{m}\right)+c_{m}^{i} D_{i} u_{m}+c_{m}^{0}=D_{i} f_{m}^{i}+g_{m} \text { in } \Omega_{m} \\
u_{m}=0 \text { on } S \Omega_{m}, u_{m}=\varphi_{m} \text { on } B \Omega_{m} .
\end{gathered}
$$

The energy inequality implies that $\left\|u_{m}\right\|_{V}$ is bounded by a constant independent of $m$. Hence, we can extract a subsequence which converges weakly in the $\|\cdot\|_{V}$ norm to a limit function $u$. The weak convergence implies that $u$ is a weak solution of (1.1), and the uniform estimate implies that (1.3) holds.

\section{Some EXtensions of the Result}

Since the boundedness of $b^{i}, c^{i}$ and $c^{0}$ is used only to verify certain estimates, we can relax the regularity of these coefficients as in [1]. Specifically, our arguments apply if

$$
\left(b^{i}\right)^{2},\left(c^{i}\right)^{2}, c^{0} \text { are in } L^{q, r} \text { and } g \in L^{q_{1}, r_{1}}
$$

for $2 / r+n / q=2, r \geq 1,2 / r_{1}+n / q_{1}=(n+4) / 2$, in which case we must replace $\|g\|_{2}$ by $\|g\|_{q_{1}, r_{1}}$. In addition, the constants $q$ and $r$ can be different for different coefficients. Finally, the constant $C$ only depends on the coefficients $b^{i}, c^{i}$, and $c^{0}$ through the norms $\left\|b^{i}-c^{i}\right\|_{q, r}$ and $\left\|\left(c^{0}\right)^{+}\right\|_{q, r}$, where $\left(c^{0}\right)^{+}$is the positive part of $c^{0}$. 
We also note that the constant $C$ can be taken independent of the length of $I(\Omega)$ under appropriate conditions. For example, if $c^{0} \leq 0$ and $b^{i}=c^{i}$, and if $g \in L^{2,1}$, then infer that

$$
\begin{aligned}
\frac{1}{2} \int_{\omega(\tau)} u^{2} d x & -\frac{1}{2} \int_{B \Omega} \varphi^{2} d x-\int_{\Omega(\tau)}\left(a^{i j} D_{j} u-f^{i}\right) D_{i} u d X \\
= & \int_{\Omega(\tau)} g u d X \leq \int_{\Omega} g u d X .
\end{aligned}
$$

Since

$$
\int_{\Omega} g u d X \leq\|g\|_{2,1}\left(\sup _{\tau} \int_{\omega(\tau)} u^{2} d x\right)^{1 / 2}
$$

we now infer that

$$
\sup _{\tau} \int_{\omega(\tau)} u^{2} d x+\int_{\Omega}|D u|^{2} d X \leq C(n, \lambda)\left[\|f\|_{2}^{2}+\|g\|_{2,1}^{2}\right]
$$

in place of (3.1), and this inequality is the appropriate analog of (1.3).

\section{REFERENCES}

1. O. A. Ladyženskaja, V. A. Solonnikov, N. N. Ural'ceva, Linear and Quasilinear Equations of Parabolic Type, American Mathematical Society, Providence, R. I., 1967. MR 39:3159b

2. P. Cannarsa, G. Da Prato, and J.-P. Zolèlsio, Evolution equations in non-cylindrical domains, Atti Accad. Naz. Lincei Cl. Sci. Fis. Mat. Natur. (8) 88 (1990), 73-77. MR 93c:35059

3. G. M. Lieberman, Regularized distance and its application, Pacific J. Math. 117 (1985), 329352. MR 87j:35101

4. G. M. Lieberman, Intermediate Schauder theory for second order parabolic equations II. Existence, uniqueness, and regularity, J. Differential Equations 63 (1986), 32-57. MR 87 m:35113b

5. J.L. Lions, Sur les problèmes mixtes pour certains systèmes parboliques dans des ouverts non cylindriques, Ann. Inst. Fourier (Grenoble) (1957), 143-182. MR 21:1455

6. Yong Jiongmin, Weak solutions of second order parabolic equations in noncylindrical domains, J. Partial Differential Equations 2 (2) (1989), 76-86. MR 90g:35068

(R. M. Brown and W. Hu) Department of Mathematics, University of Kentucky, LexINGTON, KENTUCKY 40506

E-mail address: rbrown@ms.uky.edu

E-mail address: weihu@ms.uky.edu

(G. M. Lieberman) Department of Mathematics, Iowa State University, Ames, Iowa 50011

E-mail address: lieb@iastate.edu 